\section{The industrialisation of the dental profession}

\author{
J. Cottingham ${ }^{1}$ and A. Toy ${ }^{2}$
}

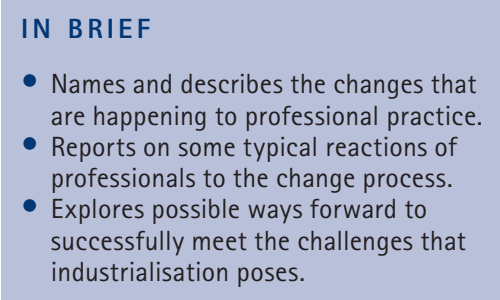

This paper arises from a dissertation submitted to the University of Nottingham towards a Masters Degree in Clinical Education. The purpose of the paper is to describe and name the process that the dental profession is experiencing. The authors will argue that this phenomenon has all the hallmarks of an industrialisation process. It will become clear that this industrialisation process is not confined to the dental profession in the UK. The key drivers for this process are described, followed by the results and analysis of a small-scale research project designed to explore the reactions of professionals to the new methods of working. Finally, there is a discussion of the implications for dental education in this new era.

\section{Introduction}

The dental profession in the UK is undergoing profound changes, perhaps the most fundamental changes it has experienced since the inception of the NHS in 1948. This has caused huge disquiet amongst the members of the profession as they sometimes struggle to adapt to these reforms. ${ }^{1,2}$

The effects of similar changes on physicians in North America have been described as 'professional decentralisation.' ${ }^{3}$ Those in the middle of their professional lives struggle most to adapt. Professionals towards the end of their working lives can retire, and for future professionals the new system is the norm.

The features of the reforms have been twofold: firstly the power to commission NHS dental care (which the majority of the public still seek) has been removed from the practitioner and taken by the state. Secondly the 'right' to

\footnotetext{
${ }_{1 *}$ GDP and Vocational Training Programme Director, Hoyle and Harris Dental Practice, 27 Dudley St, Grimsby, NE Lincolnshire, DN31 2AW; ${ }^{2}$ GDP and Faculty Board Member, Faculty General Dental Practitioners UK, Gorse Covert Dental Practice, 34 Maxwell Drive, Loughborough, Leicestershire, LE11 4RZ

Correspondence to: Dr John Cottingham Email: johnandjoyc@tiscali.co.uk
}

\section{Refereed Paper}

Accepted 11 February 2009

DOI: $10.1038 /$ sj.bdj.2009.255

${ }^{\circledR}$ British Dental Journal 2009; 206: 347-350 perform dental procedures is no longer the sole preserve of the dentist and is being devolved to other more narrowly trained operatives.

In addition to this, the professional is being instructed to practise in certain ways by government appointed agencies in order to produce outcomes to meet the aims of centrally derived protocols. These protocols are then turned into process driven targets. The days of 'full clinical freedom' are long gone. The power of the profession to define the parameters in which clinical decisions are made is under severe attack.

\section{Industrialisation}

Prior to industrial working, complex tasks were performed by skilled workers. They generally carried out all of the steps in the process of production and could mostly determine their own working methods and pace. Skills were handed down from one generation to the next and developed by experience.

Frederick Winslow Taylor is credited as the instigator of the scientific investigation of work practices. ${ }^{4} \mathrm{His}$ ideas were put into practice at the beginning of the twentieth century by such as Henry Ford in the automotive industry and led to a revolution in the efficiency of working practices. We all benefit, at least in the Western world, from that change in terms of higher standards of living and wealth.
The hallmarks of an industrialisation process are two fold: first any complicated task is broken down into simple steps; secondly each simple step is analysed to find the most efficient way of carrying out that step. ${ }^{5}$ This leads to division of labour to manage and perform each simpler step.

What are the drivers of this change? The next section outlines the influences that are pushing the process.

\section{Loss of Trust}

'All professions are a conspiracy against the laity.' (G. B. Shaw 1906)

It is a current given that society has lost trust in professional practice involved with the delivery of healthcare. The Shipman scandal, the Bristol Heart scandal and the Alder Hay Children's Hospital cases are the most commonly cited recent cases as causes of this loss of trust. Philosophers might point to a postmodern disillusionment with any form of authority, including the government, church and any institution such as the professions which claim to possess expertise and an overarching paradigm of truth. Truth held by experts is suspect. It might be used as a means of oppression.

\section{Economics}

'It's the economy stupid.' (Bill Clinton 1992 Presidential election)

Economic drivers are very powerful 
influences on human behaviour and organisation. Economics of healthcare delivery are thought to be key influences in current reforms of healthcare systems. Marcia Angell ${ }^{6}$ has coined the phrase 'double agency' to describe the changed relationship between patient and professional, that: 'economics is now driving ethics'.

Doctors are no longer acting as agents solely with the patient's best interests in mind. They also have to consider the societal costs incurred in their decisionmaking. They have: 'acquired an obligation to save resources for society.'

They act both for the patient and in the general economic interests of the community.

The success of science based medical practice has also helped to create this economic double agency. Hart $^{7}$ compares the technical and scientific advances of the nineteenth and twentieth centuries, with the parallel advances in medicine, which has been transformed into:

'a system of material production, through which an infant culture of trying based on faith, hope and mutual self deception has matured to an adult culture of verified doing, based on evidence with measured inputs, outputs and efficiency.'

Science based medicine's achievements drive patient expectations as to what is now possible, leading to everhigher proportions of national wealth being allocated to healthcare spending. What was once affordable is now thought to be unaffordable. ${ }^{8}$

\section{Simplifying complexity}

'For every complex problem there is an easy answer, and it is wrong' (H. L. Mencken)

A much more subtle influence in the industrialisation process is the push to simplify complexity and to apply standard solutions to problems, which may often be multifactorial and nuanced. One of the ways to reduce variation in practice and to standardise procedures is the creation of protocols. Although attractive there are arguments both for and against. ${ }^{9}$

On the one hand they may 'enhance the quality of care, reduce unwanted variations in practice and render medicine more scientific'.
On the other hand they may lead to: 'cookbook medicine, to deskilling and to reduce the quality of care'.

The danger is to fall into the "if A then do B' trap. This might work in many routine situations but not the complex circumstances, which clinicians face every day in their working lives. Protocols are good servants but poor masters.

Berg $^{9}$ also emphasises the perhaps unintended consequences of protocol driven medical practice:

'It is naïve to assume that the widespread use of protocols will not lead to an increasing bureaucratisation and regulation of healthcare practices... Insurance firms, governmental agencies institution management will inevitably jump on the rails laid out for them. There is no way this could not happen. Protocols are simply too similar to regulating tools that are the building stones of commercial and administrative agencies.'

The results of the influences described above have been experienced by GDPs in England and Wales since the imposition of the new contract in April 2006. This has brought NHS dental provision into line with the rest of the NHS.

The current government since 1997 has been developing the model of NHS provision of healthcare, which has been described as 'A model of medicine (which I term scientific-bureaucratic) ... that embodies many of the specific characteristics of Fordist labour processes. ${ }^{10}$

It has the following features:

1. Government appointed scientific review bodies, in healthcare, the National Institute of Clinical Excellence (NICE) examine all the available evidence on a particular aspect of healthcare delivery. Their purpose is to separate the large number of healthcare interventions which are thought to have had no proper evaluation and so be termed ineffective from effective interventions. There is also a remit to examine cost effectiveness in their decision-making

2. The recommendations are then turned into protocols and guidelines, which are disseminated to surgeries

3. The guidelines have an inherent 'if ... then' logic. Users are guided towards a particular course of action dependent upon stated prior conditions
4. The protocols and guidelines are turned into targets. Practice funding is dependent upon meeting targets and can be retrospectively withdrawn if targets are not met.

Examples of targets that general medical practitioners (GMPs) have been given to meet (with cost penalties if not met) include reduction of serum cholesterol in the population and reduction of blood pressure to a scientifically determined 'norm'. Thus we see many of our patients in dental practice taking statins and hypotensive medication.

In general dental practice, NICE guidelines on recall intervals have been recommended and antibiotic prophylaxis against bacterial endocarditis has been withdrawn. More recommendations will follow as scientific truth emerges about the effectiveness of our interventions.

\section{The professional's response to industrialisation}

We now describe the results of a smallscale research project designed to explore the professional's response to the industrialisation process. Evidence is cited from a series of in-depth unstructured interviews with professionals from varying disciplines. The interviewees included a senior partner in general medical practice, a sole owner in general dental practice, an associate general dental practitioner, a postgraduate dental educator, a senior dental nurse/tutor, and an early years teacher.

A qualitative analysis of the interview transcripts was performed using a method of 'thematic content analysis' in which the data are organised into 'emerging themes and concepts. ${ }^{11}$ In parallel with the interview analysis, literature relevant to the subject was surveyed and connections with the interview data noted and integrated into the overall analysis and conclusions.

The following themes emerged:

- Minding the machine

- Loss of autonomy

- Looking to the past

- Conflicted feelings about the role of complementary professionals

- Visioning the future.

Some themes overlap. 


\section{Minding the machine}

There was a sense that the healthcare providers are now serving a system rather than individual patients. There is more emphasis on managing the healthcare needs of the population rather than providing personal care for the individual often based on long-term relationship as well as expertise. Each member of the team involved in healthcare delivery has a more narrowly defined set of technical, reproducible tasks to perform.

There are links here to the philosophical idea of instrumentalism. You are of value to me if you can do something for me, not as a person of intrinsic worth. This can lead to people feeling like cogs in a machine.

\section{Loss of autonomy}

There was a strong feeling in the interviewees that they had lost, or were losing, autonomy in their day-to-day practice and professional lives. Government control of activity was seen as a threat to professional self-regulation and self-assessment. The old model of professionals working within parameters decided upon and policed by their peers was being severely eroded.

\section{Looking back/grieving/mourning}

Professionals were often coping with the large changes occurring in their working lives by demonising the present and glorifying the past. This relates to perceived threat to professional autonomy posed by loss of freedom and the 'threat' of paraprofessionals taking over previously protected areas of work.

\section{Conflicted feelings about complementary professionals}

In many respects the interviewees found that the help they received from staff that were not fully qualified as a teacher, doctor or dentist to be invaluable. But they also felt threatened if they perceived their role as being diminished by the employment of complementary professionals. This threat was heightened if they perceived the role of paraprofessionals as providing professional services 'on the cheap'. Interestingly the GMP was most at ease with his multiskilled team. He had the longest experience of the model, but even he felt some disquiet in extending diagnostic and prescribing rights to practice nurses.

\section{Visioning the future}

When any system undergoes major change, there are 'early adopters' who can quickly see the advantages that the new ways of working offer. There are proactive professionals, initially small in number, who have the skill to work well within the change process. The models that were anticipated (as now increasingly occurs in primary medical care) were the dentist at the hub, with many dental care professionals at the rim working as a team of mutual support.

There was also the vision of training and educational pathways branching out from traditional university based undergraduate routes. The dental care professional had a concept of a 'skills escalator' in which skills can be added to at each step. The new Peninsula Dental School has developed such a programme of education. ${ }^{12}$ By the end of year one students should be at dental nurse level, year two hygienist level, year three dental therapist level and year four dentist.

\section{Discussion}

The industrialisation process of professional practice is neither new nor confined to dentistry. While it is natural to wish to reject it, complain about it or even ignore it, the fact remains that it is part of the next chapter of dental history. The industrialisation of dentistry is creating a number of challenges to accepted thinking and practice and it's not necessarily all bad news. As the process of providing care is broken down into stages, performed by different members of the dental team, there are possible opportunities for cost savings. Greater control by government provides the mechanisms to invest resources in communities with the greatest health need. It could potentially lead to a more equitable and accessible dental service.

Applying the principle of industrialisation to a manufacturing process leads to improvements in the efficiency and consistency in production. Whilst similar improvements may be seen within the dental environment, we also have to remember that the patient is not an inanimate physical object, but a human being with the complexities of human needs and wants. The industrialisation of dentistry must also be designed to be effective, and to promote fulfilling professional careers for the team of providers. Our challenge as dental educators is to ensure that the profession is equipped to remain effective as well as efficient and consistent.

\section{What will it take to remain effective?}

In the authors' opinion, the extended dental team will require a higher level of leadership and management. Delegation of duties requires greater clarification of professional standards and values for the whole team to follow.

As portions of the care pathway are provided by multiple operators, someone must ensure that the patient's needs are accurately and clearly established at the outset. These needs must then be clearly communicated so that they don't get forgotten as they pass down the production line. This will demand greater skills in delegation and communication on the part of the dentist, whose role will be less as a producer and more as a physician. It will require great effort to see the patient as a whole person rather than a set of teeth - and as importantly - the patient must perceive that too. They will expect to feel understood as a person by every member of the dental team if they are to have trust in them.

The skills of leadership, management and communication have been identified as part of the soft skills of dentistry. ${ }^{13}$ Any educational activity to develop these skills should be structured to include not only the correct content but also the correct process to meet that need. These skills cannot be delivered via lectures but must involve small group and oneto-one teaching and mentoring activities with a high potential for self-reflection. ${ }^{14}$ Greater emphasis on team-based training is an obvious necessity.

\section{Conclusions}

Industrialisation of dentistry is happening and will remain for the foreseeable future. As a profession, we have the challenge of ensuring that the effectiveness of patient care will not suffer in 
the process of improving efficiency and consistency. There is potential for more patient-centred care, as the team work together to produce healthy patients rather than the emphasis being to correct the ravages of disease.

There will also be opportunities for those dentists who can grasp the new world by creating new models of delivery. Industrialisation requires the dental profession to speak clearly for upholding of standards of care in the changing world as no other body has the specialised professional knowledge to represent this. It is also incumbent to ensure that dentists and the whole team receive appropriate education to meet the new challenge.

1. Ward P. Happy New Year to all our readers? - and never was this more heartfelt or more significant (editorial). Br Dent J 2007; 202: 53.

2. Chestnutt | G, Thomas D R, Patel R, Treasure E T. Perceptions and attitudes to a fundamental reform of General Dental Services in Wales. Primary Dent Care 2007; 14: 13-18.

3 Castellani $B$, Wear D. Physician views on practicing professionalism in a corporate age. Qual Health Res 2000; 10: 490-506.

4. Taylor FW. The principles of scientific management New York, 1911. Published by Norton Library (1967) by arrangement with Harper \& Row Publishers.

5. Rastegar D A. Health care becomes an industry.
Ann Fam Med 2004; 2: 79.

6. Angell M. The doctor as double agent. Kennedy Inst Ethics J 1993; 3: 279-286.

7. Hart J T. Health care or health trade? A historic moment of choice. Int J Health Serv 2004; 34: 245-254.

8. Pollock A M. NHS plc: the privatisation of our health care. London: Verso, 2005.

9. Berg M. Problems and promises of protocol. Soc Sci Med 1997; 44: 1081-1088.

10. Harrison S. New labour, modernisation and the medical labour process. J Soc Policy 2002; 31: 465-485.

11. Stewart K, Gill P, Chadwick B, Treasure E. Qualitative research in dentistry. Br Dent J 2008; 204: 235-239.

12. Kay L. Personal communication to trainers meeting 28/6/07. Buxton, Derbyshire, 2007.

13. Toy A, Richards W. A specialism of primary dental care? Prim Dent Care 2004; 11: 5-7.

14. Schon D A. Educating the reflective practitioner. San Francisco: Josey-Bass, 1987 